\title{
Research on electric vehicle load forecasting based on travel data
}

\author{
Xu Lin ${ }^{1, *}$, Wang Bing ${ }^{1}$, Cheng Mingxi ${ }^{1}$ and Fang Shangshang ${ }^{1}$ \\ ${ }^{1}$ College of Energy and Electrical Engineering, Hohai University, Nanjing 211199, China
}

\begin{abstract}
Due to the rapid promotion of electric vehicles, large-scale charging behavior of electric vehicles brings a large number of time and space highly random charging load, which will have a great impact on the safe operation of distribution network. This paper proposes a planning method of electric vehicle charging station based on travel data. Firstly, the didi trip data is processed and mined to get the trip matrix and other information. Then, the electric vehicle charging load forecasting model is established based on the established unit mileage power consumption model and charging model, and the charging demand distribution information is predicted by Monte Carlo method. Finally, the simulation analysis is carried out based on the trip data of some areas of a city, which shows the effectiveness of the established model feasibility.
\end{abstract}

\section{Introduction}

In the process of modernization of China's governance system and governance capacity, "green water and green mountains" is the foundation. In order to reduce the harm caused by traditional fuel vehicles to the environment, the state has vigorously developed the electric vehicle industry through a series of policies. Electric vehicles have become an important part of the development of new energy in the country. Compared with fuel vehicles, electric vehicles can be derived from wind power and hydropower generation, and realize the function of energy saving and emission reduction. Therefore, it has been highly valued by the government and widely concerned by the society. With the development of society, the scale of electric vehicles is also increasing. The charging behavior of large-scale EV seriously affects the safe operation of the power grid, so it is critical to accurately predict the vehicle charging load ${ }^{[1-5]}$.

At present, there are many research results about charging station planning. In reference[6], the electric vehicles for different purposes are divided, and the parking probability model is generated to simulate the starting and stopping times and state of electric vehicles in a day. The charging load of EV in a certain area is simulated by Monte Carlo method, and then the temporal and spatial distribution of charging load is obtained. In reference[7], the influence of EV load on local distribution network under different charging modes is compared and analyzed by using Monte Carlo method and probability statistical model. The paper[8] constructs simple and complex chains, establishes EV driving time-space distribution model, simulates the driving behavior of electric vehicles with different permeability and no date type by Monte Carlo method, obtains the charging demand and predicts the charging load curve. reference[9] by establishing EV state transfer model, the paper analyzes the choice of different travel chains and destinations of EV users, describes the correlation between different space positions of electric vehicles by using correlation function, and analyzes the uncertainty of EV travel by using the system state estimation method. The deficiency of the above research lies in that this kind of method relies on a large number of data to obtain the probability model, which will not work in the absence of data; moreover, the actual travel rules of various regions are quite different, and the results obtained by using other regions to represent the region are not accurate.

In this paper, through the open platform of didi Gaia data, we get the travel data of didi in Chengdu, which can well reflect the travel rules of the city residents. By processing and mining the data, we get the OD matrix and trajectory matrix. Then, according to the influence of temperature and road grade on vehicle power consumption, we establish the unit mileage power consumption model. Then, we consider the EV charging characteristics and different charging methods The EV charging model is established. Based on the above two models, the EV charging load forecasting model is established and solved by Monte Carlo method, and the temporal and spatial distribution of EV load is obtained. Finally, the effectiveness of the proposed model is verified by a city data simulation.

*Corresponding author: 15850686503@163.com 


\section{Travel data processing and mining}

\subsection{Data display and processing}

The vehicle travel data comes from the "Gaia open data set" of didi Gaia data open plan. The data obtained are the vehicle data of Chengdu Second Ring Road from November 1 to 30,2016, including order data and track data. The data attributes are shown in Table 1:

Table 1. Data attribute

\begin{tabular}{|c|c|c|}
\hline Type & Field & $\begin{array}{l}\text { Field } \\
\text { type }\end{array}$ \\
\hline \multirow{5}{*}{$\begin{array}{l}\text { Order } \\
\text { data }\end{array}$} & Order ID & Varchar \\
\hline & Start billing time & $\begin{array}{c}\text { Datatim } \\
\mathrm{e}\end{array}$ \\
\hline & End billing time & $\begin{array}{c}\text { Datatim } \\
\mathrm{e}\end{array}$ \\
\hline & Location Longitude & Double \\
\hline & Location latitude & Double \\
\hline \multirow{5}{*}{$\begin{array}{l}\text { Trajecto } \\
\text { ry data }\end{array}$} & Vehicle ID & Varchar \\
\hline & Order ID & Varchar \\
\hline & Instantaneous time & $\begin{array}{c}\text { Datatim } \\
\mathrm{e}\end{array}$ \\
\hline & Instantaneous longitude & Double \\
\hline & Instantaneous latitude & Double \\
\hline
\end{tabular}

It can be seen from table 1 that the real-time position and time of the vehicle can be obtained directly from the trajectory data, and the time and position of the boarding point and alighting point can be obtained from the order data ${ }^{[10]}$. Because people use a lot of taxi software such as didi when they travel, the data obtained is more in line with the actual situation of travel.

In order to eliminate the interference of noise data, this paper deals with the data as follows:

(1) Delete the data whose travel distance is less than 500 meters;

(2) Delete the data whose origin and destination are not in the specified area.

At the same time, because the travel data provided by didi uses the Mars coordinate system (GCJ-02) for positioning, and the coordinate system of the geographic information software used in this paper is WGS-84. In order to avoid deviation, coordinate system transformation is needed in coordinate projection ${ }^{[11]}$.

\subsection{Data mining}

Due to the randomness of EV charging load in time and space, the origin destination analysis method is used to fully explore the spatial distribution characteristics of EV charging load. The core of this method is to construct OD matrix. The OD matrix and trajectory matrix $T$ are obtained by mining the processed data.

(1) Trajectory mapping

In the processed track data, the longitude and latitude coordinates and time of the same order with a time interval of 2-4 seconds are extracted to form a track matrix $T$. The track data points corresponding to each order are shown in formula (1):

$$
T_{i}=\left\{\left(x_{i}^{1}, y_{i}^{1}, t_{i}^{1}\right),\left(x_{i}^{2}, y_{i}^{2}, t_{i}^{2}\right), \cdots,\left(x_{i}^{n}, y_{i}^{n}, t_{i}^{n}\right)\right\}
$$

Where, $T_{i}$ is the trajectory data set of the $i$-th order, $i$ is the number of orders, $\left(x_{i}^{1}, y_{i}^{1}, t_{i}^{1}\right)$ is the latitude and longitude position and time of the $i$-th order start point, $\left(x_{i}^{2}, y_{i}^{2}, t_{i}^{2}\right)$ is the latitude and longitude position and time of the $i$-th order middle time, and $\left(x_{i}^{n}, y_{i}^{n}, t_{i}^{n}\right)$ is the latitude and longitude position and time of the $i$-th order end point.

(2) Establish OD matrix

By extracting the trajectory data of the start and end points corresponding to all orders in the trajectory $\operatorname{set} T$, the OD point distribution set can be obtained as follows:

$$
\begin{gathered}
O=\left\{\left(x_{1}^{1}, y_{1}^{1}, t_{1}^{1}\right),\left(x_{2}^{1}, y_{2}^{1}, t_{2}^{1}\right), \cdots,\left(x_{i}^{1}, y_{i}^{1}, t_{i}^{1}\right)\right\} \\
D=\left\{\left(x_{1}^{n}, y_{1}^{n}, t_{1}^{n}\right),\left(x_{2}^{n}, y_{2}^{n}, t_{2}^{n}\right), \cdots,\left(x_{i}^{n}, y_{i}^{n}, t_{i}^{n}\right)\right\}
\end{gathered}
$$

Where $O$ is the set of track points at the starting point and $D$ is the set of track points at the ending point.

\section{Power consumption model of unit mileage}

There are many factors that affect the power consumption per unit mileage, but the traffic condition and temperature lead to the largest proportion of additional power consumption.

(1) Traffic energy consumption model

Under different road conditions, the individual driving behavior of car owners will lead to different unit energy consumption ${ }^{[12]}$. According to the code for design of urban road engineering, the urban roads are divided into four grades. According to the measured traffic data, the power consumption model of unit mileage is established by equation (4):

$$
\varepsilon_{l}^{t}=\sum_{n=0}^{3} a_{n}\left(v_{l}^{t}\right)^{n-1}
$$

Where, $\varepsilon_{l}^{t}$ is the power consumption per unit mileage under $l$ road level, $l=\{1,2,3,4\} ; v_{l}^{t}$ is the speed at time $t$ under level $l ; a_{n}$ is the EV energy consumption factor under different road levels.

(2) Temperature energy consumption model

Based on the user travel records collected by a company in North America, the general functional relationship between EV power consumption per mileage and ambient temperature is obtained, as shown in equation (5):

$$
\phi_{\text {Tet }}=\sum_{i=0}^{5} b_{n}\left(1.8 T_{e t}+32\right)^{n}
$$

Where, $\phi_{T e t}$ is the unit mileage power consumption under different temperatures, $T_{e t}$ is the ambient temperature, and $b_{n}$ is the temperature energy consumption factor.

(3) Comprehensive energy consumption model 
When the electric vehicle runs on the road, it will be affected by the traffic road grade and temperature at the same time, so it is necessary to combine the two in modeling. According to the temperature energy consumption model shown in equation (5), when the temperature is $20^{\circ} \mathrm{C}$, the power consumption of $\mathrm{EV}$ is the lowest. Therefore, based on $\phi_{20}$, the power consumption ratio $k_{T e t}$ when the temperature $T_{e t}$ is defined as:

$$
k_{T e t}=\frac{\phi_{T e t}}{\phi_{20}}
$$

Where $\phi_{20}$ is the unit mileage power consumption at $20^{\circ} \mathrm{C}$ and $\phi_{T_{e t}}$ is the unit mileage power consumption at $T_{e t}{ }^{\circ} \mathrm{C}$.

Considering the influence of traffic and temperature, the unit mileage power consumption $\varepsilon^{t}$ of temperature and traffic can be calculated by equation (7).

$$
\varepsilon^{t}=\varepsilon_{l}^{t} k_{\text {Tet }}
$$

\section{EV charging model}

\subsection{Charging pile model}

According to "conductive charging interface for electric vehicles" (QC / T 841-2010), this paper classifies charging facilities into slow charging (L1), conventional charging (L2) and fast charging (L3). The charging power of L1 charging pile is $3.5 \mathrm{~kW}$, that of L2 charging pile is $7 \mathrm{~kW}$, and that of L3 charging pile is $50 \mathrm{~kW}$. Because of the level of charging power, L1 and L2 charging piles are AC charging piles, while L3 charging pile is DC charging pile.

\subsection{EV charging characteristics}

The charging characteristics of EV include SOC at the beginning of trip, SOC at the end of charge and charging duration, which will directly affect the temporal and spatial distribution of charging load.

(1) Initial trip SOC

The initial SOC of EV determines the duration of the vehicle, which will directly affect the size of the charging load. In this paper, according to the reference ${ }^{[13]}$, the SOC at the initial travel time conforms to the normal distribution $N(0.7,0.1)$, and the probability density function is shown in equation (8):

$$
f(s)=\frac{10}{\sqrt{2 \pi}} \exp \left(-50(x-0.7)^{2}\right)
$$

(2) Charging demand calculation

The state of charge of the $i$ th EV at any time during the mileage is shown in equation (9):

$$
S^{T}(i)=S^{0}(i)-\sum_{j=1}^{T} l_{j-1, j} \varepsilon^{t}(i)
$$

Where, $S^{T}(i)$ is the state of charge of the $i$ th EV at time $T, S^{T}(i)$ is the state of charge at the initial time, $l_{j-1, j}$ is the distance from time $j-1$ to time $j$ in the trajectory matrix $T$, and $\mathrm{F} \varepsilon^{t}(i)$ is the power consumption per unit mileage.

1) Private car charging demand judgment

This type of vehicle carries out power supplement after arriving at the destination. If the current power $S^{T}(i)$ is not enough to support arriving at the destination, it triggers the charging demand and finds the nearest charging station to supplement power

$$
S^{T}(i) \leq l_{T, n} \varepsilon^{t}(i)
$$

2) Taxi charging demand judgment

$$
S^{T}(i) \leq S_{\varepsilon}
$$

Where $S_{\varepsilon}$ is the user mileage anxiety coefficient, which obeys the uniform distribution between 0.15 and 0.3 . If this condition is triggered during the journey of carrying passengers, turn to equation (9) for judgment; otherwise, judge again.

3) Judgment of charging demand of utility vehicles

This kind of car is mainly used for office commuting. If its power can meet the demand of mileage, it will charge slowly at the destination, otherwise it will trigger the charging demand immediately.

(3) End of charge SOC

The SOC after the end of charging can directly affect the time distribution of charging load. In this paper, the SOC after the end of charging conforms to the normal distribution $N(0.8,0.2)$, and its probability density function is shown in equation (12):

$$
f(s)=\frac{20}{3 \sqrt{2 \pi}} \exp \left(-\frac{200(x-0.7)^{2}}{9}\right)
$$

(4) Charging time calculation

Since EV will choose fast charging or slow charging according to the remaining situation of electric quantity, and the charging power between fast and slow charging piles is different, the charging time of the a EV is calculated according to the different charging piles. The charging time of the $i \mathrm{EV}$ is $T_{s}(i)$ :

$$
T_{s}(i)=\frac{S^{A C}(i)-S^{n}(i)}{\eta_{c} * P_{k}} C_{\text {battery }}(i)
$$

Where $S^{A C}(i)$ is SOC after the charging of the $i$ th EV, $S^{n}(i)$ is SOC of the $i$ th EV at time $n, C_{\text {battery }}(i)$ is the battery capacity of the $i$ th $\mathrm{EV} ; \eta_{c}$ is the charging efficiency of charger, $\eta_{c}=0.9 ; P_{k}$ is the type of charging pile.

(5) Charging demand calculation

The total load $P_{c}$ in the area is:

$$
\begin{gathered}
P_{c}=\sum_{j=1}^{n e} \sum_{k=1}^{24} P_{k} \mu_{j}(k) \\
\mu_{j}(k)=\left\{\begin{array}{l}
1, t_{i}^{n} \leq T_{i} \leq t_{i}^{n}+T_{s}(i) \\
0, \text { other }
\end{array}\right.
\end{gathered}
$$

Where ne represents the total number of electric vehicles in the area and $\mu_{j}(k)$ indicates whether the $i$ th $\mathrm{EV}$ is charged at time $k$. 


\subsection{Electric vehicle load forecasting model}

After the above model is established, figure 1 gives the specific flow chart of the distribution of the charging load time-space prediction model. When judging the charging demand, if charging demand is generated, the location information and residual SOC of charging demand point are stored in the information matrix of charging demand, and the required charge amount is added to the load curve. After the cycle is over, the charging load curve can be obtained.

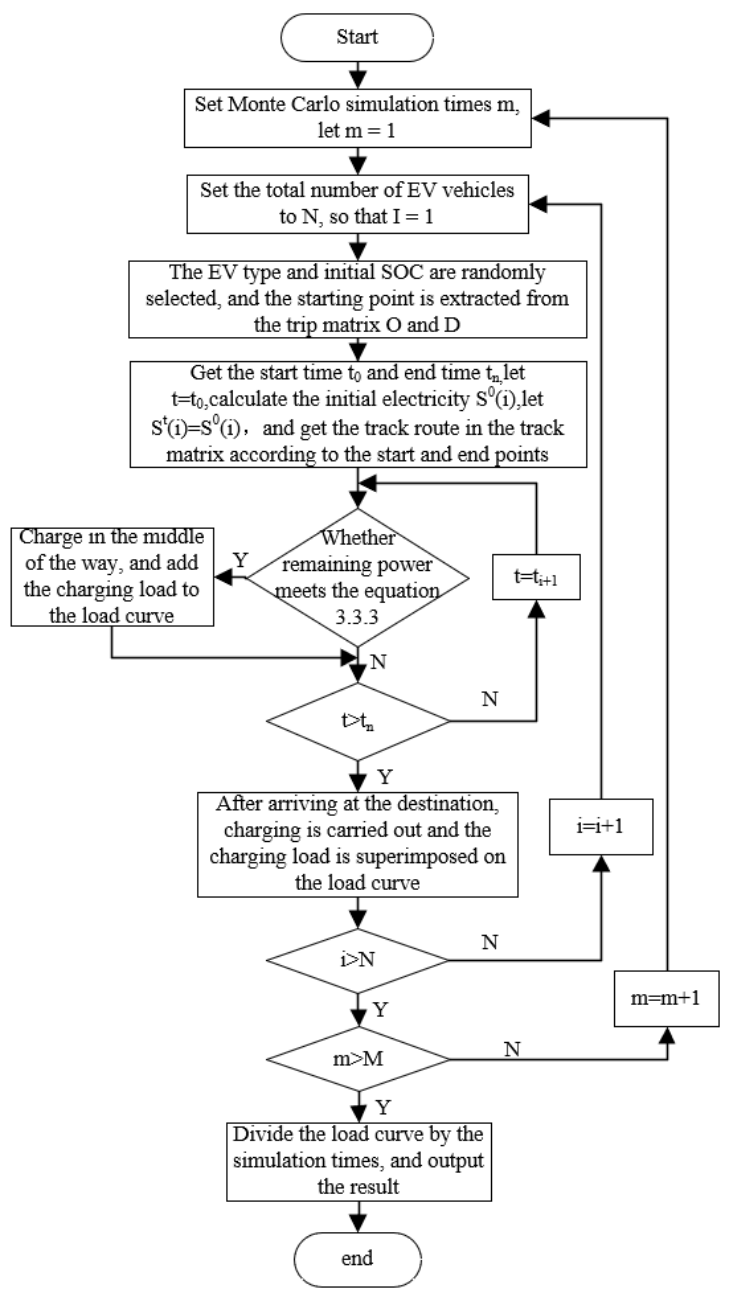

Figure 1. Load forecasting process

\section{Example analysis}

\subsection{Simulation parameter setting}

The planned area of Chengdu is about $77 \mathrm{~km}^{2}$, with 10000 EV, 5000 private cars, 3000 taxis and 2000 official cars. The types of electric vehicles widely existing in the market are shown in Table 2. It can be seen that the mileage of the electric vehicles is generally over $300 \mathrm{~km}$. The types of private cars and public cars in this paper are randomly selected from the table. Because taxi drivers prefer to consider a long mileage, for taxi types, the eu5-r500, BAIC Ex5 and BYD E5 with a endurance of more than $400 \mathrm{~km}$ are selected.
Table 2. Electric vehicle model parameters

\begin{tabular}{|c|c|c|}
\hline Car type & $\begin{array}{c}\text { Battery } \\
\text { capacity }\end{array}$ & $\begin{array}{c}\text { Driving range } \\
\text { of NEDC }\end{array}$ \\
\hline BYD ev360 & 43.2 & 305 \\
\hline BYD E5 & 51.2 & 405 \\
\hline BAIC eu5-r500 & 53.6 & 416 \\
\hline BAIC Ex5 & 61.8 & 415 \\
\hline Geely Dihao & 41 & 298 \\
\hline Roewe erx5 & 48 & 317 \\
\hline BAIC ev160 & 26 & 150 \\
\hline
\end{tabular}

\subsection{Analysis of simulation results}

By sampling 10000 electric vehicles with Monte Carlo method, the OD matrix and trajectory matrix $T$ obtained from didi travel data mining are used to allocate travel data for each electric vehicle. After travel simulation, the charging demand information matrix $M$ and $\mathrm{EV}$ charging load data can be obtained. After drawing the EV charging load data into a curve, it is shown as follows:

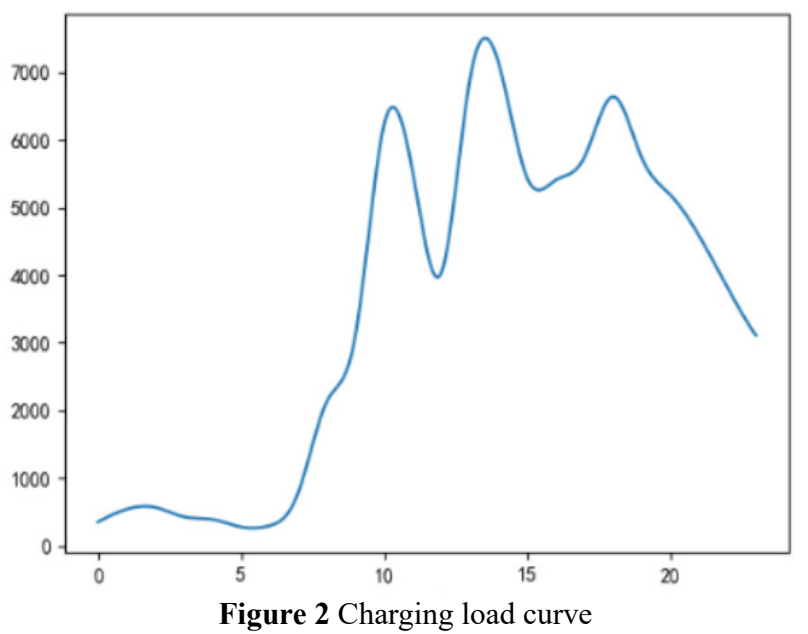

From Figure 2, it can be seen that the red places represent the more vehicles need to be charged here, while the red part is distributed in the lower left corner, and the red range in the upper right corner is small, because the lower left corner is the traffic volume in the central area of the city, and the traffic volume in the upper left corner is the small suburban traffic of the city. At the same time, it can be seen from Figure 4 that the load curve reaches the peak at about 10:00, 14:00 and 18:00. After people arrive at the unit by driving, they will charge and supplement the electric energy due to mileage anxiety. After lunch break and meal activities arrive at the unit, the electric energy will be replenished generally.

\section{Conclusion}

In this paper, the problem of electric vehicle charging load forecasting is studied, and the electric vehicle charging load forecasting model based on didi travel data and its solution method are proposed. Firstly, by mining didi travel data, the travel OD matrix and trajectory matrix are obtained. Then, considering the factors of temperature and road grade, the unit mileage power consumption model is established, and the EV charging model is established 
Monte Carlo method is used to solve the model, and the results of load forecasting are obtained.

\section{References}

1. Frade I, Ribeiro A, Goncalves G, Antunes A P, Transportation Research Record: Journal of Transportation Research Board. 2552, 91-98 (2011)

2. CHEN Lidan, ZHANG Yao, Antonio FIGUEIREDO, Power System Protection And Control.41, 140148(2014)

3. XING Hao, FU Minyue, LIN Zhiyun, IEEE Transactions on Power Systems, 31(5), 41184127(2016)

4. WU Juai, XUE Yusheng, XIE Donglaing, Automation of Electric Power Systems, 42(13), 101-107(2018)

5. CHEN Lidan, NIE Yongquan, ZHONG Qing, Transactions of China Electrotechnical Society, 30(4), 216-225(2015)
6. SHI Shaunglong, JIA Zhuo, Power System Technology. 34(11), 126-130(2010)

7. SUN Xiaoming, WANG Wei, SU Su, Electric Power Automation Equipment, 39(3), 207-213 (2019)

8. ZHANG Cong, XU Xiaohui, SUN Haishun, Power System Protection And Control. 14, 29-34(2014)

9. Yongquan N, CHUNG C. Y., XU N. Z, IEEE Transactions on Power Systems, 31(6), 46054615(2016)

10. ZhANG Chengjia, LIU Junyong, XIANG Yue, Proceedings of the CSEE. 38, 1054-1064 (2018)

11. TIAN Liting, SHI Shuanglong, JIA Zhuo, Power System Protection and Control. 44, 17-23(2016)

12. SONG Yunong, LIN Shunjiang, TANG Zhiqiang, Automation of Electric Power Systems. 44, 4762(2020)

13. LIU Wenxia, LONG Rishang, XU Xiaobo, Automation of Electric Power Systems. 40, 45-52 (2016) 